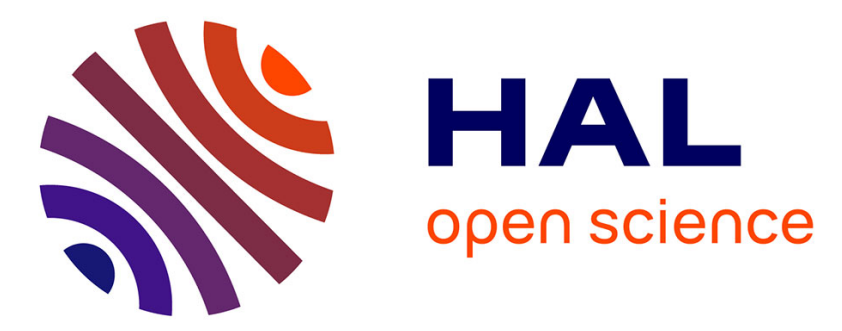

\title{
Dis moi ce que tu penses de la propriété intellectuelle, je te dirai qui tu es
}

Rémy Guichardaz, Thomas Coudert, Herrade Igersheim-Chauvet, Mathieu

Lefebvre, Julien Pénin

\section{- To cite this version:}

Rémy Guichardaz, Thomas Coudert, Herrade Igersheim-Chauvet, Mathieu Lefebvre, Julien Pénin. Dis moi ce que tu penses de la propriété intellectuelle, je te dirai qui tu es. Revue Economique, 2019, 70, pp.22. 10.3917/reco.706.1213 . hal-02435554

\section{HAL Id: hal-02435554 https://hal.science/hal-02435554}

Submitted on 10 Jan 2020

HAL is a multi-disciplinary open access archive for the deposit and dissemination of scientific research documents, whether they are published or not. The documents may come from teaching and research institutions in France or abroad, or from public or private research centers.
L'archive ouverte pluridisciplinaire HAL, est destinée au dépôt et à la diffusion de documents scientifiques de niveau recherche, publiés ou non, émanant des établissements d'enseignement et de recherche français ou étrangers, des laboratoires publics ou privés. 


\title{
«Dis-moi ce que tu penses de la propriété intellectuelle, je te dirai qui tu es ${ }^{*} *$
}

\author{
Rémy Guichardaz ${ }^{1}$, Thomas Coudert ${ }^{2}$, Herrade Igersheim ${ }^{3}$, Mathieu \\ Lefebvre $^{4}$, Julien Pénin 5 \\ Université de Strasbourg, Université de Lorraine, CNRS, \\ BETA, F-67000 Strasbourg, France
}

\begin{abstract}
Résumé. L'objet de cet article est d'analyser de manière expérimentale les liens supposés entre les préférences sociales des individus et la manière dont ils conçoivent la propriété intellectuelle, en opposant en particulier les approches utilitaristes et déontologiques. Pour ce faire, nous avons mis en place un protocole expérimental original à l'interface de deux courants de littérature : l'un, bien établi, traitant des préférences sociales ; l'autre, naissant, tentant de capter les différentes justifications de l'existence de la propriété intellectuelle. Notre expérience aboutit à deux enseignements majeurs : 1) les sujets utilitaristes semblent changer de conception de la justice sociale dès lors que la question de la propriété intellectuelle est posée; 2) les sujets rawlsiens sont en parfaite cohérence avec l'approche déontologique de la propriété intellectuelle, donnant la priorité aux droits des auteurs et des inventeurs, tout en minimisant les inégalités pouvant en résulter en optant, par exemple, en faveur d'une durée courte de propriété intellectuelle.
\end{abstract}

JEL Codes : C 91 ; D 63 ; 034

Mots-clefs. Préférences sociales, économie expérimentale, Rawls, propriété intellectuelle

* Les auteurs tiennent à remercier les éditeurs de la Revue Economique ainsi que les deux rapporteurs pour leurs commentaires et remarques constructives.

\footnotetext{
${ }^{1}$ Contact : rguichardaz@unistra.fr

${ }^{2}$ Contact : t.coudert@unistra.fr

${ }^{3}$ Contact : igersheim@unistra.fr

${ }^{4}$ Contact : mathieu.lefebvre@unistra.fr

${ }^{5}$ Contact : penin@unistra.fr
} 


\section{Introduction}

L'analyse économique standard justifie l'existence de la propriété intellectuelle par l'incitation économique (Arrow, 1962). En effet, les biens immatériels partagent en partie les caractéristiques de biens publics induisant ainsi une défaillance de marché que la propriété intellectuelle a pour objectif de pallier. En accroissant les incitations à investir dans la production de biens immatériels la propriété intellectuelle permet de «réajuster» la production desdits biens au niveau socialement optimal. La propriété intellectuelle est donc justifiée par ses conséquences (en termes d'incitation pour les créateurs, en termes de répercussion sur le bien-être social etc.). On parle alors d'une vision conséquentialiste ou utilitariste de la propriété intellectuelle (Merges, 2016). Dans une telle optique, le système de propriété intellectuelle se justifie en ce qu'il augmenterait l'utilité totale de la société en encourageant les activités inventives et créatives.

Cette justification utilitariste de la propriété intellectuelle s'oppose à une justification alternative, dite déontologique : selon celle-ci, la propriété intellectuelle est perçue comme juste en soi et ses conséquences ne sont considérées que dans un second temps. Pour nourrir son plaidoyer en faveur d'une justification déontologique de la propriété intellectuelle, Merges (2011) invoque notamment Emmanuel Kant ou encore John Rawls. En effet, la théorie de la justice de John Rawls (1971) s'inscrit dans une perspective déontologique et se présente explicitement comme une alternative à l'utilitarisme $(1971$, p. 3) : c'est elle que nous retiendrons dans cet article en tant que représentante de l'approche déontologique ${ }^{6}$.

On le voit, ces deux principales manières de justifier la propriété intellectuelle (utilitarisme versus déontologie) sont chacune fondées sur des conceptions divergentes de la justice sociale. Partant de cela, notre étude a pour ambition d'interroger plus avant ces liens supposés entre conception de la justice sociale d'un côté, conception de la propriété intellectuelle de l'autre grâce à l'économie expérimentale. Ainsi, à la lumière du débat académique entre approches déontologique et utilitariste de la propriété intellectuelle, on pourrait en effet s'attendre à ce que les individus exprimant des préférences sociales de type déontologique aient une conception de la propriété intellectuelle distincte de ceux ayant des préférences sociales utilitaristes. En effet, les arguments mobilisés par l'une et l'autre approche ne sont pas de même nature: dans le cas de l'utilitarisme, les institutions ne sont justifiées qu'en raison des conséquences qu'elles génèrent sur le bien-être social. A l'inverse, dans le cas de l'approche déontologique rawlsienne, les institutions sont d'abord justifiées par l'existence de justes procédures. Dans cet article, nous cherchons à déterminer si ces liens supposés entre les préférences sociales des individus et leur conception de la propriété intellectuelle sont vérifiés empiriquement ou pas en adoptant une démarche expérimentale.

Pour construire notre protocole expérimental, nous invoquons deux types de littérature jusqu'alors disjoints - la littérature expérimentale traitant des préférences sociales d'une part,

\footnotetext{
${ }^{6}$ Rappelons que la théorie de la justice rawlsienne ne saurait être réduite au seul critère du maximin. Faire preuve d'une conception rawlsienne de la justice va au-delà d'un choix qui maximise les gains des individus les plus défavorisés et comprend une attention réelle aux justes procédures et au fait que les individus sont porteurs de droits inaliénables.
} 
et celle traitant de la propriété intellectuelle d'autre part. D'un côté, le courant s'intéressant à la question des préférences sociales a d'ores et déjà donné lieu à une abondante littérature (voir la synthèse récente de Cooper et Kagel, 2016). Les préférences sociales véhiculent l'idée que les choix des individus ne sont pas seulement dictés par leur propre bien-être ou gain, mais dépendent également des niveaux de bien-être ou de gains d'autrui. Dans des expérimentations mettant en scène le jeu de l'ultimatum, le jeu du dictateur ou encore le jeu de bien public, ces travaux ont mis au jour un certain nombre d'éléments permettant d'expliquer pourquoi les choix individuels s'écartent de la rationalité économique standard : altruisme, réciprocité, aversion pour l'iniquité, etc. (voir la synthèse proposée par Lewitt et List, 2007). De même, dans des jeux de distribution simples, l'enjeu est de déterminer la raison de la préférence d'un individu pour une distribution plutôt qu'une autre - distribution efficiente qui maximise le gain collectif, distribution qui maximise le gain du plus défavorisé, distribution qui est la plus équitable... -, l'individu étant alors soit perçu comme un « architecte social », son gain n'étant pas affecté par son choix (Engelmann et Strobel, 2004), soit confronté au «voile d'ignorance » rawlsien, c'est-à-dire ne connaissant pas le rôle et donc le gain qui lui reviendra une fois son choix de distribution ou de société effectué (Johansson-Stenman, Carlsson et Daruvala 2002, Calrsson, Gupta et Johansson-Stenmann 2003). C'est ce dernier type d'approche que nous privilégions dans ce travail.

De l'autre côté, la littérature expérimentale relative à la propriété intellectuelle compte quelques travaux très récents qui soit examinent la validité de l'argument de l'incitation (Meloso 2009, Buchanan et Wilson 2014, Bruggemann et al. 2015, Engel et Kleine 2015), soit s'intéressent à la perception que les individus ont de la propriété intellectuelle, en recourant notamment à des questionnaires (Mandel 2015, Mandel et al. 2015, Fast et al. 2016). Nous nous inspirons de cette seconde catégorie de travaux pour notre protocole. En outre, une troisième catégorie de littérature se doit d'être mentionnée compte tenu de la nature de notre recherche, notamment axée sur la déontologie : il s'agit de celle s'intéressant à la procedural fairness. En termes expérimentaux, il a en effet été démontré que les individus sont sensibles à cet aspect (Bolton et al. 2005, Krawczyk and Le Lec 2010, Brock et al. 2013, Linde and Sonnemans 2015, Kurz, Orland et Posadzy 2018), tandis que certains modèles théoriques (Trautmann 2009, Krawczyk 2011, Saito 2013) en rendent également compte en intégrant une forme d'attention aux procédures dans les fonctions d'utilité individuelles.

Au regard de notre question de recherche - les liens supposés entre les préférences sociales, utilitaristes ou déontologiques, des individus et leur conception de la propriété intellectuelle -, nos résultats mettent en avant deux enseignements principaux: 1) les sujets utilitaristes semblent changer de conception de la justice sociale dès lors que la question de la propriété intellectuelle est posée ; 2) les sujets déontologiques rawlsiens semblent davantage cohérents, donnant la priorité aux droits des auteurs et des inventeurs, tout en minimisant les inégalités pouvant en résulter en optant, par exemple, en faveur d'une durée courte de propriété intellectuelle.

Avant de poursuivre, précisons que notre travail n'a pas pour objectif de fournir de nouveaux arguments en faveur ou en défaveur de la propriété intellectuelle, et notamment de savoir si celle-ci incite effectivement les individus à davantage innover. En outre, notre étude n'a pas 
de visée normative en ce qu'elle ne tente pas de déterminer si la propriété intellectuelle devrait être, dans nos sociétés contemporaines, effectivement fondée sur des considérations utilitaristes ou déontologiques.

La section suivante présente notre protocole expérimental. La troisième section exposera les résultats qui seront discutés dans une quatrième section. La cinquième section proposera quelques remarques conclusives.

\section{Protocole expérimental}

Notre protocole expérimental a pour objet de confronter les préférences sociales des individus à leur conception de la propriété intellectuelle. Pour ce faire, nous avons mis en place un protocole comportant trois étapes auxquelles participent 24 joueurs. Principalement basée sur un jeu de distribution simple proposé par Engelmann et Strobel (2004), la première étape a pour but d'identifier les conceptions de la justice sociale endossées par les joueurs en fonction de leur préférence pour l'une des trois distributions proposées : égalitariste (E), rawlsienne (R) ou utilitariste $(\mathrm{U})^{7}$. Les 24 joueurs sont ici répartis aléatoirement en 8 groupes de 3 joueurs et sont confrontés successivement à 4 matrices de distributions, sachant que les groupes sont reformés à chacune d'elles de telle manière à ce que les joueurs ne peuvent avoir connaissance de l'identité des autres joueurs de leur groupe, ni a fortiori de leurs décisions. Ces matrices sont proposées aux joueurs de manière aléatoire afin d'éviter tout biais d'ancrage. Un exemple de matrice de distributions est proposé dans le tableau 1.

Tableau 1. Exemple de matrice de distributions de l'étape 1

\begin{tabular}{|c|c|c|c|}
\hline & Société A & Société B & Société C \\
\hline Joueur 1 & 21 & 28 & 15 \\
\hline Joueur 2 & 12 & 12 & 12 \\
\hline Joueur 3 & 7 & 4 & 6 \\
\hline Total & 40 & 44 & 33 \\
\hline
\end{tabular}

Ainsi, si un joueur fait le choix de la société ou de la distribution A, il sera identifié comme étant rawlsien puisqu'il s'agit de la société qui maximise le gain de l'individu le plus défavorisé (ici, le joueur 3 qui reçoit 7 dans la société A contre 4 en B et 6 en C). Si un joueur fait le choix de la société B, il sera identifié comme étant utilitariste puisqu'il s'agit cette fois de la société qui maximise l'utilité collective (comprise ici comme correspondant au gain total ; en effet, le gain total de la société B est de 44, contre 40 en A et 33 en B). Enfin, si un joueur fait le choix de la société $\mathrm{C}$, il sera identifié comme étant égalitariste, étant donné que la société $\mathrm{C}$ minimise les écarts de gains entre les 3 individus ${ }^{8}$. Les sujets ignorent leur rôle dans le jeu au moment du choix, rôle qui leur sera attribué par la suite de manière aléatoire

\footnotetext{
${ }^{7}$ Suivant Engelmann et Strobel (2004) et même si ceci n'est pas au cœur de notre question de recherche, nous avons introduit ici une conception égalitariste de la justice afin de couvrir un spectre plus large de préférences sociales.

${ }^{8}$ La société C satisfait les deux définitions habituelles de l'équité : la première, proposée par Fehr et Schmidt (1999), fait l'hypothèse que les individus réprouvent la différence entre leur gain et ceux des autres ; la seconde de Bolton et Ockenfels (2000) considère que la distribution la plus équitable pour les individus est celle qui minimise la différence entre leur gain et le gain moyen.
} 
afin de les rétribuer. Aucune indication concernant les probabilités d'être désigné joueur 1, 2 ou 3 n'est fournie, reproduisant peu ou prou par-là le célèbre « voile d'ignorance » rawlsien.

La deuxième étape de notre protocole comporte 4 matrices de distributions successivement proposées à des groupes de trois joueurs modifiés à chacune d'elles, mais inclut à présent la propriété intellectuelle. Il est cette fois demandé aux sujets de choisir entre deux distributions ou deux sociétés : l'une où la propriété intellectuelle est reconnue et où le joueur créateur, supposé être le joueur 3, tire bénéfice de ses activités inventives ; l'autre qui ne reconnaît pas la propriété intellectuelle et où le joueur 3 n'est pas privilégié par rapport aux deux autres malgré son investissement créatif. Un exemple de matrice de distributions pour cette deuxième étape est proposé dans le tableau 2.

Tableau 2. Exemple de matrice de distributions de l'étape 2

\begin{tabular}{|l|c|c|}
\hline & Société A & Société B \\
\hline Joueur 1 & 6 & 11 \\
\hline Joueur 2 & 16 & 16 \\
\hline Joueur 3 & 20 & 19 \\
\hline Total & 44 & 46 \\
\hline Commentaire & $\begin{array}{c}\text { Dans cette société, le joueur 3 } \\
\text { ateur/inventeur est protégé par } \\
\text { un droit de propriété } \\
\text { intellectuelle. }\end{array}$ & $\begin{array}{c}\text { Dans cette société, il n'y a } \\
\text { pas de PI. Le joueur 3 } \\
\text { auteur/inventeur ne reçoit } \\
\text { aucune protection légale de } \\
\text { ses œuvres/inventions }\end{array}$ \\
\hline
\end{tabular}

Comme celles du tableau 2, les trois autres matrices de l'étape 2 sont agencées de telle sorte à ce que les sujets soient censés privilégier la société sans propriété intellectuelle. En effet, si les sujets sont insensibles à tout raisonnement déontologique s'agissant de la propriété intellectuelle, alors ils devraient tous sans exception privilégier la société B puisqu'elle est tout à la fois celle qui maximise le revenu de l'individu le plus défavorisé (joueur 1), qui maximise l'utilité collective et qui est la plus égalitaire. A l'inverse, des individus privilégiant la société A témoigneraient du fait que ces derniers adoptent un raisonnement déontologique s'agissant de la propriété intellectuelle, c'est-à-dire un raisonnement donnant la priorité au respect des justes procédures.

Enfin, une troisième étape clôt notre protocole expérimental par un questionnaire déclaratif relatif à la conception de la propriété intellectuelle des participants ${ }^{9}$. Ce dernier inclut en outre des questions sociodémographiques ainsi que des questions de contrôle sur le risque. Remis suite à cette troisième étape, les gains des joueurs sont déterminés de la façon suivante : l'une des 8 matrices de distributions est tirée au sort et le gain réalisé par les joueurs pour celle-ci est son gain pour l'expérience (le facteur de conversion est de un euro pour un point).

\section{Résultats}

\footnotetext{
${ }^{9}$ Ledit questionnaire est reproduit en annexe 4.
} 
Nous avons réitéré l'expérimentation à 4 reprises auprès de 96 étudiants de diverses disciplines de l'Université de Strasbourg début juillet 2018. La présentation des résultats se fera en deux temps. Il convient tout d'abord d'utiliser les matrices de distributions de la première étape afin de catégoriser les sujets selon leur conception de la justice sociale : rawlsien, utilitariste ou égalitariste. Dans un second temps, nous exposons les résultats de la deuxième étape relatifs à la prise de position desdits sujets pour ou contre la propriété intellectuelle. Il s'agit enfin de déterminer dans quelle mesure les préférences sociales des individus perdurent dès lors qu'ils sont confrontés à la question de la propriété intellectuelle.

Afin d'agréger l'information recueillie lors de la première étape et en tirer une classification cohérente, nous utilisons la méthode de l'Analyse des Correspondances Multiples (ACM par la suite). Le but de la méthode est de synthétiser plusieurs informations qualitatives relatives à chaque individu, permettant ainsi une catégorisation plus aisée. Chaque sujet peut ainsi être représenté dans un espace à plusieurs dimensions, chaque dimension correspondant à une information collectée en première étape. Le nuage de point ainsi obtenu, est lisible, est ensuite résumé à partir des deux ou trois axes qui permettent d'expliquer la majorité des différences entre sujets. L'analyse peut alors se concentrer sur les dimensions principales qui résument les variations observées ${ }^{10}$. Dans le cas de notre étude, cette méthode a également l'énorme avantage de pouvoir trancher entre deux types de préférences qui, en fonction des choix, pourraient s'appliquer à un même individu. En effet, les choix observés ne permettent pas toujours d'identifier une préférence claire.

Les deux premières lignes du tableau 3 présentent la classification des sujets de l'expérience selon l'ACM. Nous observons que la majorité d'entre eux ont des préférences sociales soit rawlsiennes $(34,38 \%)$, soit utilitaristes $(35,42 \%)$, tandis qu'une proportion plus faible fait preuve de préférences sociales égalitaristes (18,75\%). Malgré l'usage de l'ACM pour classer nos sujets, il subsiste une indétermination pour un certain nombre de sujets, catégorisés en tant que «autres ». Pour ces derniers, la méthode ne nous permet pas de trancher à ce stade entre des préférences égalitaristes ou rawlsiennes. Nous reviendrons par la suite sur les choix spécifiques de ce groupe.

Sur base de la classification des sujets selon leur conception de justice sociale, nous pouvons maintenant examiner si cette dernière est corrélée avec leur choix en faveur ou en défaveur de la propriété intellectuelle lors de l'étape 2. Plus spécifiquement, nous essayons de déterminer si les préférences révélées à l'étape 1 sont corrélées ou non avec le choix fait à l'étape 2 pour ou contre un régime de propriété intellectuelle. Dans le tableau 3, on observe que de façon générale une plus grande proportion de rawlsiens $(50,8 \%)$ et d'utilitaristes $(47,8 \%)$ choisissent la société qui protège la propriété intellectuelle. Parmi les égalitaristes, seuls $41,7 \%$ la choisissent et 34,1\% dans la catégorie « autres ». Ces résultats globaux sont cohérents avec les choix observés pour chaque matrice prise individuellement, à l'exception de la catégorie «autres ». En effet, vu le faible nombre de sujets, il est difficile à ce stade de tirer des conclusions (en particulier, le fait qu'un individu seulement de cette catégorie choisisse la société avec propriété intellectuelle pour la $2^{\text {ème }}$ matrice de distributions). Nous avons

\footnotetext{
${ }^{10}$ Le détail de la méthodologie et les résultats intermédiaires figurent en annexe.
} 
également testé s'il y avait des différences de choix entre les catégories et nous n'observons pas de différence significative ${ }^{11}$.

Tableau 3. Classification des sujets selon leur préférence sociale et choix en faveur de la propriété intellectuelle par catégorie

\begin{tabular}{lcccc}
\hline & \multicolumn{4}{c}{ Proportion de sujets selon leur préférence } \\
\cline { 2 - 5 }$\#$ & Égalitaristes & Rawlsiens & Utilitaristes & Autres \\
\cline { 2 - 5 }$\%$ & 18 & 33 & 34 & 11 \\
& $18,75 \%$ & $34,38 \%$ & 35,42 & $11,45 \%$ \\
\hline \multicolumn{5}{c}{ Choix de la société A selon la catégorie de sujets } \\
Total & Égalitaristes & Rawlsiens & Utilitaristes & Autres \\
\cline { 2 - 5 }$(\%)$ & 30 & 67 & 65 & 15 \\
Matrice 1 & $(41,7)$ & $(50,8)$ & $(47,8)$ & $(34,1)$ \\
$(\%)$ & 8 & 15 & 18 & 4 \\
Matrice 2 & $(44,5)$ & $(45,5)$ & $(52,9)$ & $(36,4)$ \\
$(\%)$ & 10 & 20 & 18 & 1 \\
Matrice 3 & $(55,6)$ & $(60,6)$ & $(52,9)$ & $(9,1)$ \\
$(\%)$ & 7 & 19 & 13 & 5 \\
Matrice 4 & $(38,9)$ & $(57,6)$ & $(38,2)$ & $(45,5)$ \\
$(\%)$ & 5 & 13 & 16 & 5 \\
\hline & $(27,8)$ & $(39,4)$ & $(47,1)$ & $(45,5)$ \\
\hline
\end{tabular}

Ces premiers résultats ont cependant le défaut de ne pas tenir compte de l'hétérogénéité possible au sein de notre population de sujets, hétérogénéité qui pourrait expliquer leurs choix. Nous poursuivons donc notre examen par une analyse de régression des choix de société (société A ou B) dans laquelle nous contrôlons pour une série de facteurs potentiellement importants.

Le tableau 4 présente les résultats de deux types de régression. Premièrement, nous expliquons par une régression linéaire le nombre de fois où les sujets ont choisi la société protégeant la propriété intellectuelle au cours des quatre décisions prises à l'étape 2 (société A). Ensuite, nous regardons les facteurs explicatifs du choix entre la société A et la société B pour l'ensemble des observations au moyen d'une régression Probit dans laquelle nous corrigeons les écarts-types pour tenir compte de la répétition des observations pour un même sujet. Les variables explicatives dans chacune des régressions sont la conception de la justice sociale endossée par les sujets en étape 1, l'âge, le sexe, le type d'études suivies, les préférences politiques $^{12}$, la préférence auto-déclarée pour le risque ${ }^{13}$, ainsi que des contrôles relatifs au comportement et à l'appréciation des sujets face à la propriété intellectuelle.

\footnotetext{
${ }^{11}$ Le Tableau A3 dans l'annexe présente les résultats des tests de Mann-Whitney de différence de choix entre nos catégories de sujet. Seule la catégorie «autres » qui rassemble des sujets pour lesquels on ne peut trancher entre des préférences égalitaristes ou rawlsiennes présente une différence significative de choix avec les rawlsiens. Cela tendrait à faire pencher la balance vers une catégorisation de ces sujets dans le groupe des égalitaristes.

${ }^{12}$ Il a été demandé aux sujets d'évaluer leur position politique sur un axe politique de 0 (extrême gauche) à 100 (extrême droite).

${ }^{13} \mathrm{Il}$ a été demandé aux sujets d'évaluer leur préférence pour le risque sur une échelle de 0 à 10 (voir Vieider et al., 2015).
} 
Tableau 4. Analyse de régression des choix de société

\begin{tabular}{ccc}
\hline & MCO & Probit \\
Variables dépendantes : & \# de choix A & Choix A \\
Préférences sociales : & $(1)$ & $(2)$ \\
Constante (Egalitaristes) & -0.790 & $-2.034^{* * *}$ \\
Rawlsiens & $(1.348)$ & $(0.657)$ \\
Utilitaristes & $0.613^{* *}$ & $0.419^{* * *}$ \\
Autres & $(0.270)$ & $(0.155)$ \\
& $0.448^{*}$ & $0.321^{* * *}$ \\
Age & $(0.268)$ & $(0.159)$ \\
Male & 0.023 & 0.024 \\
Caractéristiques démographiques: & $(0.370)$ & $(0.200)$ \\
Questions relatives à la PI : & $0.049^{* * *}$ & $0.040^{* * *}$ \\
Q2 : « La PI est justifiée » & $(0.023)$ & $(0.015)$ \\
& $0.509^{* *}$ & $0.370^{* * *}$ \\
Q1 : « Durée de la PI + longue » & $(0.219)$ & $(0.150)$ \\
& & \\
N & 0.671 & $0.463^{* * *}$ \\
& $(0.436)$ & $(0.152$ \\
& $-0.212^{* *}$ & $-0.145^{* * *}$ \\
& $(0.089)$ & $(0.052)$ \\
\hline
\end{tabular}

Notes : Les écarts-types sont présentés entre parenthèses. Toutes les régressions contrôlent également le type d'étude suivie, les préférences politiques telles que renseignées dans le questionnaire de fin d'expérience, de la préférence auto-déclarée pour le risque exprimée en variables binaires, ainsi que d'un contrôle si le sujet a déjà téléchargé du contenu illégalement. Les écarts-types des régressions probit sont corrigés par cluster pour tenir compte de la répétition des observations par sujet.

Dans le tableau 4, on voit donc que le nombre de fois où les sujets ont choisi la société avec propriété intellectuelle est significativement plus élevé chez les sujets rawlsiens, tandis que l'effet est marginalement significatif chez les utilitaristes. Lorsque l'on regarde la probabilité de choisir la société avec propriété intellectuelle, elle est plus grande chez les rawlsiens et les utilitaristes: ces deux catégories de sujets choisissent donc de manière significative une société avec propriété intellectuelle. Quant aux sujets égalitaristes et «autres », il n'y a pas de différence entre eux pour les cas (1) et (2).

Nous pouvons retirer de ce tableau quelques enseignements supplémentaires. Tout d'abord, nous observons un lien significatif entre le fait de vieillir et d'être en faveur de la propriété intellectuelle confirmant par-là les résultats de Mandel (2015ab). Nous pouvons de plus noter que les hommes sont davantage en faveur de la propriété intellectuelle que les femmes. Bien que non renseigné dans le tableau $6^{14}$, on observe que les sujets ayant exprimé une préférence élevée pour le risque (c'est-à-dire supérieure à 5 sur une échelle de 0 à 10) sont également davantage favorables à la propriété intellectuelle. En revanche, contrairement aux travaux de Mandel, aucun lien significatif n'est observé entre l'orientation politique ou le type d'études suivies et une préférence en faveur de la propriété intellectuelle. La variable de contrôle la plus intéressante pour notre question de recherche correspond ici à la première question de

\footnotetext{
${ }^{14}$ Voir le tableau en annexe 2 pour les résultats complets.
} 
notre questionnaire relative à la durée souhaitée de la propriété intellectuelle (Q1). Il a été demandé aux sujets de noter sur une échelle de -2 à +2 leur préférence pour une propriété intellectuelle plus courte ou plus longue par rapport à la durée actuelle ${ }^{15}$, la valeur 0 correspondant au statu quo. Les résultats montrent une relation significative entre le fait de déclarer préférer une durée plus courte de la propriété intellectuelle par rapport au niveau actuel et le fait de choisir la société avec propriété intellectuelle. De manière fort intéressante, ce résultat est essentiellement déterminé par les sujets rawlsiens, comme le montre la figure 1. Autrement dit, les sujets rawlsiens, qui affichent la préférence la plus forte pour une société avec propriété intellectuelle, sont également ceux qui manifestent une préférence en faveur d'une propriété intellectuelle courte. Le test de Mann-Whitney confirme que les sujets rawlsiens et les sujets utilitaristes fournissent des réponses significativement différentes à la question Q1 (cf. Tableau en annexe 3).

Figure 1. Appréciation de la durée de la propriété intellectuelle par catégorie de sujets sur une échelle de -2 à +2 (Question Q1)

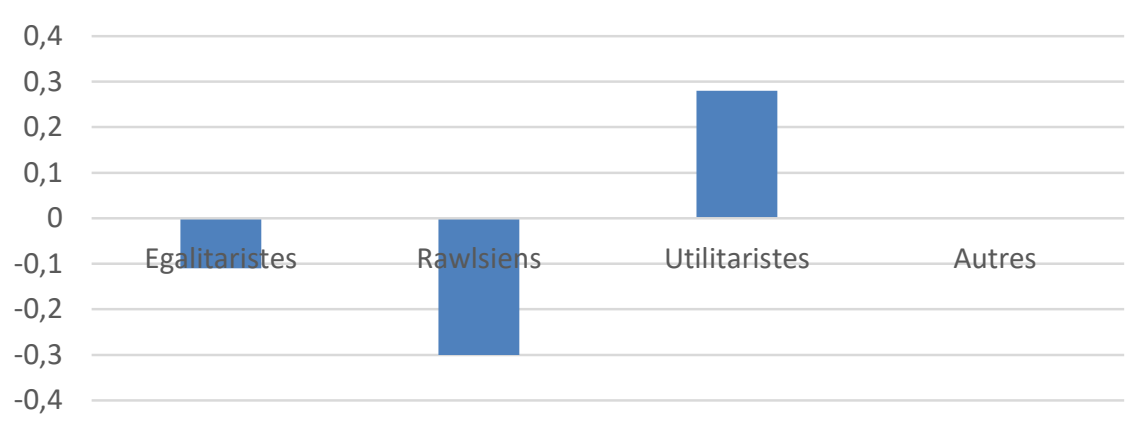

Pour résumer, ces analyses montrent qu'il existe de fortes corrélations entre les conceptions de la justice sociale affichées par les sujets et leur positionnement en faveur ou en défaveur de la propriété intellectuelle: i) les sujets utilitaristes privilégient la société avec propriété intellectuelle alors même que celle-ci ne maximise pas le gain total, ii) Les sujets rawlsiens privilégient encore plus fortement que les sujets utilitaristes la société avec propriété intellectuelle même si celle-ci ne maximise pas le gain de l'individu le plus défavorisé. De plus, les sujets rawlsiens sont significativement en faveur d'une durée courte de propriété intellectuelle. iii) Enfin, même si ces derniers n'étaient pas au cœur de notre étude, nous avons pu relever que les sujets égalitaristes tendent à privilégier une société sans propriété intellectuelle qui est, dans notre protocole, effectivement la société la moins inéquitable d'après les critères de Fehr et Schmidt (1999) et Bolton et Ockenfels (2000).

\section{Discussion}

Nos analyses mettent en avant deux résultats centraux : d'une part, nos résultats offrent une justification frappante de la propriété intellectuelle en termes déontologiques, les sujets rawlsiens donnant la priorité aux droits des auteurs et des inventeurs, tout en minimisant les inégalités pouvant en résulter en optant en faveur d'une durée courte de propriété

\footnotetext{
${ }^{15}$ La durée légale actuelle de la propriété intellectuelle est de 20 ans pour les brevets et de l'ensemble de la durée de vie de l'auteur plus 70 ans après sa mort pour les œuvres littéraires et artistiques.
} 
intellectuelle. En effet, il a été dit en introduction que l'approche déontologique - ici, illustrée par la théorie rawlsienne - donnait priorité au respect des règles, et ce avant d'envisager les conséquences desdites règles. De fait, nous constatons bien que les sujets rawlsiens n'optent pas pour la société sans propriété intellectuelle, alors que celle-ci maximise le gain de l'individu le plus défavorisé, mais pour la société avec propriété intellectuelle qui, elle, respecte les droits du créateur. Cela tend à montrer que ces sujets considèrent ces droits comme étant fondamentaux et leur donnent priorité par rapport à la distribution des gains qu'ils génèrent.

En outre, les rawlsiens ont significativement indiqué leur préférence pour une durée de propriété intellectuelle courte, signifiant par-là qu'ils sont également attentifs, mais dans un second temps, ainsi que le veut l'approche rawlsienne, à la question des conséquences ou, autrement dit, à l'effectivité des règles et droits concomitants. Certes, des inégalités peuvent advenir de par la propriété intellectuelle, mais elles doivent être minimisées afin d'aboutir à une société juste.

D'autre part, les individus affichant en première étape des préférences sociales utilitaristes défendent une société avec propriété intellectuelle alors que cette dernière ne maximise pas les gains totaux, offrant par-là un résultat délicat à interpréter. Nos résultats montrent que ces sujets changent de conception dès lors que la question de la propriété intellectuelle est posée. Cela indiquerait que faisant fi de leur volonté de maximiser l'utilité collective, les utilitaristes face à la propriété intellectuelle deviendraient attentifs à la question de la procédure ou de la règle avant celle des conséquences.

Il convient cependant de préciser que ce dernier résultat peut également s'expliquer par certaines limites de notre protocole expérimental. En particulier, le fait de considérer que les préférences sociales de l'étape 1 ont un pouvoir prédictif quant aux choix susceptibles d'être faits par les sujets lorsqu'ils sont confrontés à des matrices différentes lors de l'étape 2 peut être interrogé. Nous avons ainsi supposé que l'étape 1 permet d'éliciter des «conceptions de la justice », et pas seulement de connaître les préférences des sujets entre les différentes distributions proposées. Dès lors, à l'effet de contexte lié à la propriété intellectuelle créé en étape 2 - dont découlent nos résultats - pourrait s'ajouter un éventuel effet dû au changement des matrices de distribution entre les étapes 1 et 2 . Nous soutenons toutefois que si ce dernier effet existe, il est probablement faible : d'une part, les matrices des deux étapes reflètent les mêmes préférences sociales (rawlsienne, utilitariste, égalitariste) dans des schèmes de distribution raisonnablement similaires ; d'autre part, supposer l'existence d'un effet de ce type ne permet pas d'expliquer la cohérence et la significativité des résultats observés chez les individus rawlsiens.

Une autre façon, peut-être plus convaincante, de rendre compte du comportement surprenant des utilitaristes serait de questionner la typologie effectuée en étape 1. Dans cette perspective, ce n'est pas tant la pertinence de cette typologie que son interprétation même qui poserait problème : les sujets considérés comme étant utilitaristes pourraient être envisagés comme des sujets adoptant une conception déontologique non-rawlsienne de la justice selon laquelle tout travailleur à un droit naturel de s'approprier l'intégralité de son travail. Cette conception 
«lockéenne $»^{16}$ ou «méritocratique » de la justice expliquerait aussi bien les choix de ces individus en étape $1^{17}$ qu'en étape 2 et pourquoi ils favorisent, in fine, des droits de propriété plus longs.

\section{Remarques conclusives}

Dans cette étude, la première du genre à notre connaissance, nous avons confronté les préférences sociales des individus à leur positionnement en faveur ou en défaveur de la propriété intellectuelle. Pour ce faire, nous avons mis au point un protocole expérimental original à l'interface de deux courants de littérature : l'un, bien établi, traitant des préférences sociales ; l'autre, naissant, tentant de capter la question des incitations au cœur de la propriété intellectuelle ainsi que la perception des individus à son sujet.

Nous avons ainsi montré qu'il existait des liens indéniables entre les préférences sociales des individus et leur façon de justifier la propriété intellectuelle : d'une part, les sujets rawlsiens sont cohérents en ce qu'ils privilégient tout à la fois une société avec propriété intellectuelle tout en tâchant de minimiser les inégalités susceptibles d'en résulter. D'autre part, les sujets utilitaristes semblent basculer vers un raisonnement déontologique dès lors que la question de la propriété intellectuelle est posée en renonçant à choisir la société la plus efficace ; enfin, même si ce n'était pas là au cœur de notre questionnement, les sujets égalitaristes optent en faveur d'une société sans propriété intellectuelle, la moins inégalitaire.

Nos deux premiers résultats semblent conforter la théorie de Merges selon laquelle il existe une «grammaire morale universelle» en faveur de la propriété intellectuelle. Pour lui, «IP rules rely on a set of moral judgments that are so common and pervasive that they are close to being universal » (Merges, 2017, p. 695). Au-delà, notre étude met en avant l'existence de multiples manières de penser la propriété intellectuelle, certaines étant moins «économiques » que d'autres. La coexistence de ces différentes façons de rationaliser la propriété intellectuelle offre une éventuelle explication aux nombreuses controverses contemporaines qui l'entourent. En effet, rappelons que la propriété intellectuelle reste difficile à valider empiriquement. ${ }^{18}$. Du point de vue utilitariste, de tels résultats peuvent militer en faveur d'une réforme substantielle de la propriété intellectuelle, voire, de sa suppression (Boldrine \& Levine, 2008). A l'inverse, pour l'approche déontologique, la propriété intellectuelle demeure un droit inaliénable qui ne saurait être entièrement remis en cause au nom de la maximisation du bien-être total.

\footnotetext{
${ }^{16}$ La théorie lockéenne fonde la propriété sur le travail de l'individu créateur et reste l'une des théories déontologiques les plus utilisées aujourd'hui pour justifier la propriété intellectuelle (Moore, 2001 ; Merges, 2011 ; Mossoff, 2012).

${ }^{17}$ Selon cette interprétation, ces individus pourraient envisager la société égalitariste et rawlsienne de l'étape 1 comme une version altérée (via une politique de redistribution) de la société utilitariste qui, elle, représenterait la société émanant d'un « ordre naturel » récompensant chacun selon ses mérites.

${ }^{18}$ Voir par exemple l'étude de Lerner (2009) et les calculs de Bessen et Meurer suggèrent que « it is unlikely that patents today are an effective policy instrument to encourage innovation overall » (2008, p. 216). Plus récemment, Williams affirme qu'il y a « no credible empirical evidence on the seemingly simple question of whether stronger patent rights - either longer patent terms or broader patent rights - encourage research investments [...]» (2017, p. 28). Voir aussi la synthèse réalisée par de Beer (2016) aboutissant aux mêmes conclusions.
} 
Ce travail devra naturellement être prolongé de multiples manières afin d'approfondir et de nuancer nos résultats. Par exemple, un jeu expérimental mettant véritablement les individus en situation d'inventeur ou de créateur pourrait nous en dire davantage sur l'attitude pro- ou anti-propriété intellectuelle, ces derniers pouvant choisir en amont la société avec ou sans propriété intellectuelle dans laquelle ils souhaiteraient vivre. Une autre piste de recherche intéressante à poursuivre serait également d'établir des liens plus forts avec la littérature théorique et/ou expérimentale sur la procedural fairness.

\section{Références}

Arrow, K. J. (1962), "Economic welfare and the allocation of resources for invention" In The Rate and Direction of Inventive Activity : Economic and Social Factors, Princeton Universty Press, pp. 609-626. Palgrave, London.

Bessen, J., Meurer, M. J. (2008), Patent failure: How judges, bureaucrats, and lawyers put innovators at risk. Princeton University Press.

Boldrin, M., \& Levine, D. K. (2008), Against intellectual monopoly, (Vol. 78). Cambridge: Cambridge University Press.

Bolton, G. E., Brandts, J., \& Ockenfels, A. (2005). "Fair procedures: Evidence from games involving lotteries". Economic Journal, 115(506), 1054-1076.

Bolton, G. Ockenfels, A. (2000), "ERC: a theory of equity, reciprocity and competition", American Economic Review, 90(1), pp. 166-193.

Brock, J. M., Lange, A., \& Ozbay, E. Y. (2013). Dictating the risk: Experimental evidence on giving in risky environments. American Economic Review, 103(1), 415-437.

Brüggemann, J., Crosetto, P., Meub, L., Bizer, K. (2016), “Intellectual property rights hinder sequential innovation. Experimental evidence", Research Policy, 45(10), 2054-2068.

Buchanan, J. A., Wilson, B. J. (2014), “An experiment on protecting intellectual property”, Experimental Economics, 17(4), 691-716.

Carlsson, F., Gupta, G., Johansson-Stenman, O. (2003), "Choosing from behind a veil of ignorance in India”, Applied Economics Letters, 10(13), 825-827.

Cooper, D. J., Kagel, J. H. (2016), "Other regarding preferences: A selective survey of experimental results", To appear in the Handbook of Experimental Economics, 2. Princeton University Press.

de Beer, J. (2016), "Evidence-Based Intellectual Property Policymaking: An Integrated Review of Methods and Conclusions", The Journal of World Intellectual Property, 19(5-6), 150-177.

Engel, C., \& Kleine, M. (2015), "Who is afraid of pirates? An experiment on the deterrence of innovation by imitation", Research Policy, 44(1), 20-33.

Engelmann, D., Strobel, M. (2004), "Inequality aversion, efficiency and maximin preferences in simple distribution experiments", American Economic Review, 94(4), pp. 857-869. 
Fast, A. A., Olson, K. R., Mandel, G. N. (2016), "Experimental investigations on the basis for intellectual property rights", Law and human behavior, 40(4), 458.

Fehr, E., Schmidt, K.M. (1999), “A theory of fairness, competition and cooperation", Quarterly Journal of Economics, 114(3), pp. 817-868.

Johansson-Stenman, O., Carlsson, F., Daruvala, D. (2002), "Measuring future grandparents preferences for equality and relative standing", The Economic Journal, 112(479), 362-383.

Krawczyk, M. (2011). A model of procedural and distributive fairness. Theory and Decision, $70(1), 111-128$.

Krawczyk, M., \& Le Lec, F. (2010). 'Give me a chance!' An experiment in social decision under risk. Experimental Economics, 13(4), 500-511.

Kurz, V., Orland, A. and Posadzy, K. (2018), "Fairness versus efficiency: how procedural fairness concerns affect coordination”, Experimental Economics 21(3): 601-626.

Lerner, J. (2009), “The empirical impact of intellectual property rights on innovation: Puzzles and clues", American Economic Review, 99(2), 343-48.

Lewitt, S. D., List, J. A. (2007), "What do laboratory experiments measuring social preferences reveal about the real world?", Journal of Economic perspectives, 21(2), 153-174.

Linde, J., \& Sonnemans, J. (2015). Decisions under risk in a social and individual context: The limits of social preferences? Journal of Behavioral and Experimental Economics, 56, 62-71.

Mandel, G. N. (2015a), "The Public Perception of Intellectual Property", Florida Law Review, Vol. 66, 261.

Mandel, G. N., Fast, A. A., Olson, R. (2015b), “Intellectual property Law's Plagiarism Fallacy", BYU L. Rev., 915.

Meloso, D., Copic, J., \& Bossaerts, P. (2009), "Promoting intellectual discovery: patents versus markets", Science, 323(5919), 1335-1339.

Merges, R. P. (2011), Justifying intellectual property, Harvard University Press.

Merges, R. P. (2016), Philosophical Foundations of IP Law: The Law and Economics Paradigm, UC Berkeley Public Law Research Paper No. 290713.

Merges, R. P. (2017), “Against utilitarian fundamentalism”, St. John's Law Review, Vol. 90, No. 3.

Moore. A. D. (2001), Intellectual Property and Information Control: Philosophic Foundations and Contemporary Issues, Transaction Publishing, PDF Draft.

Mossoff, A. (2012), "Saving Locke from Marx: The labor theory of value in intellectual property theory", Social Philosophy and Policy, 29(2), 283-317. 
Rawls, J. (1971), A theory of justice, Harvard University Press, Cambridge.

Saito, K. (2013). Social preferences under risk: Equality of opportunity versus equality of outcome. American Economic Review, 103(7), 3084-3101.

Trautmann, S. T. (2009). A tractable model of process fairness under risk. Journal of Economic Psychology, 30(5), 803-813.

Vieider, F. M., Lefebvre, M., Bouchouicha, R., Chmura, T., Hakimov, R., Krawczyk, M., Martinsson, P. (2015), "Common components of risk and uncertainty attitudes across contexts and domains: Evidence from 30 countries", Journal of the European Economic Association, 13(3), 421-452.

Williams, H. L. (2017), « How Do Patents Affect Research Investments? », Annual Review of Economics, 2017 9:1, 441-469.

\section{Annexe 1 : L'analyse par composantes multiples}

Afin d'étudier l'impact des préférences sociales des individus sur leur comportement vis-à-vis de la propriété intellectuelle, il convient tout d'abord de déterminer si les réponses des individus aux quatre premières matrices font qu'ils adoptent un comportement plutôt « utilitariste », plutôt « rawlsien » ou plutôt « égalitariste ». Pour répondre à cette question et afin de déterminer si un individu adopte un comportement en particulier, nous avons utilisé la méthodologie de l'Analyse des Correspondances Multiples (ACM par la suite).

Pour réaliser cette ACM, nous avons tout d'abord identifié quatorze comportements différents concernant les réponses des individus aux quatre premières matrices. Par exemple, un premier comportement type est celui d'un individu qui aurait eu zéro réponse associable à un comportement égalitariste, deux réponses associables à un comportement rawlsien et deux réponses associables à un comportement utilitariste. Ces réponses sont alors centrées-réduites.

Les résultats de l'ACM sont résumés dans les tableaux et les graphiques suivants

Tableau A1. Dimensions de l'ACM et valeurs propres.

\begin{tabular}{ccc}
\hline Valeur propre & $\begin{array}{c}\text { Variance } \\
\text { (pourcentage) }\end{array}$ & $\begin{array}{c}\text { Variance cumulative } \\
\text { (pourcentage) }\end{array}$
\end{tabular}




\begin{tabular}{l|ccc} 
Dimension 1 & $1.63 \mathrm{e}+00$ & $5.44 \mathrm{e}+01$ & 54.44 \\
Dimension 2 & $1.37 \mathrm{e}+00$ & $4.56 \mathrm{e}+01$ & 100.00000 \\
Dimension 3 & $5.42 \mathrm{e}-20$ & & $1.81 \mathrm{e}-18$
\end{tabular}

Fig. A1 Graphique du cercle des corrélations

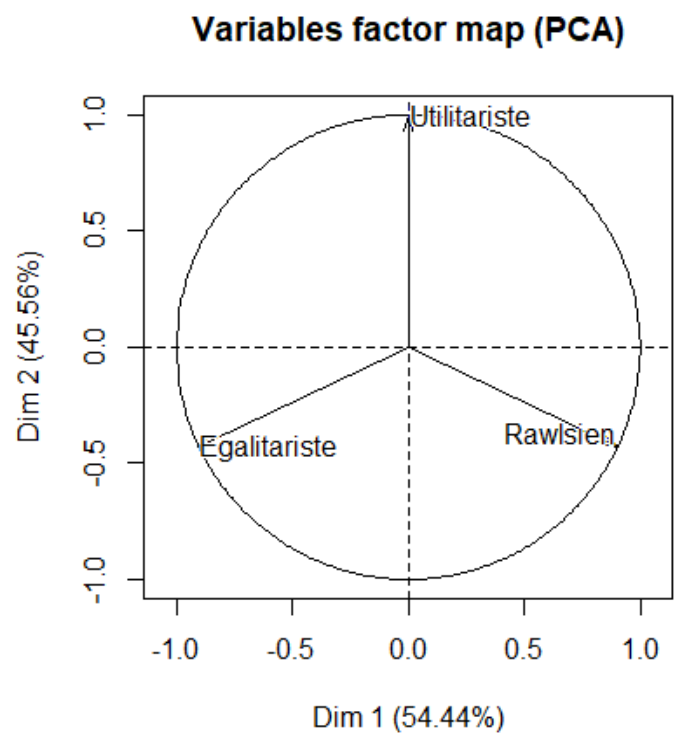

Nous pouvons tout d'abord constater que les valeurs propres de la première dimension et de la deuxième dimension sont supérieures à 1 , ce qui implique que les composantes principales ainsi proposées permettent une bonne représentation des variances. Notons également que la troisième dimension n'apporte aucune information supplémentaire (sa valeur propre tendant vers zéro).

L'ACM nous a ainsi permis ainsi de regrouper les quatorze comportements différents selon deux dimensions: une première dimension qui permet de déterminer si un individu a un comportement rawlsien ou égalitariste et une seconde dimension qui permet de déterminer si un individu a ou n'a pas un comportement utilitariste.

Compte tenu de ces deux dimensions, l'ACM nous permet partiellement de catégoriser les différents comportements des individus. Les résultats sont ainsi résumés dans la fig. A2 et le tableau A2.

Fig A2. Comportements types et dimensions de l'ACM. 


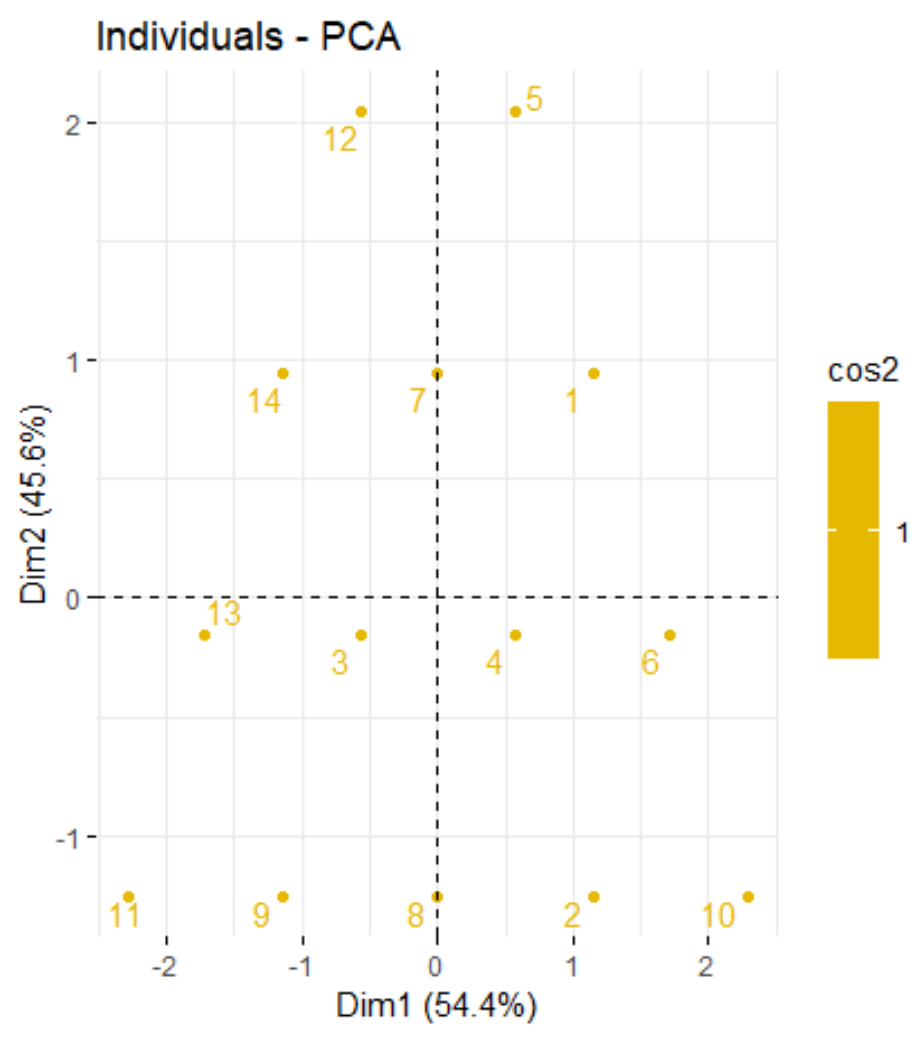

Tableau A2. Les comportements types (centrés-réduits) des individus et les conclusions à la suite de l'ACM

\begin{tabular}{l|cccl}
\hline Comportement & Égalitariste & Rawlsien & Utilitariste & Conclusion \\
\hline 1 & $-1,1127$ & 0,4451 & 0,7796 & Utilitariste \\
2 & $-0,3338$ & 1,2240 & $-1,0395$ & Rawlsien \\
3 & 0,4451 & $-0,3338$ & $-0,1299$ & Égalitariste \\
4 & $-0,3338$ & 0,4451 & $-0,1299$ & Rawlsien \\
5 & $-1,1127$ & $-0,3338$ & 1,6892 & Utilitariste \\
6 & $-1,1127$ & 1,2240 & $-0,1299$ & Rawlsien \\
7 & $-0,3338$ & $-0,3338$ & 0,7796 & Utilitariste \\
8 & 0,4451 & 0,4451 & $-1,0395$ & Indéterminé \\
9 & 1,2240 & $-0,3338$ & $-1,0395$ & Égalitariste \\
10 & $-1,1127$ & 2,0029 & $-1,0395$ & Rawlsien \\
11 & 2,0029 & $-1,1127$ & $-1,0395$ & Égalitariste \\
12 & $-0,3338$ & $-1,1127$ & 1,6892 & Utilitariste \\
13 & 1,2240 & $-1,1127$ & $-0,1299$ & Égalitariste
\end{tabular}


Note : Le Tableau A.2 se lit de la façon suivante : la première colonne renvoie à un comportement type observé à partir des réponses de l'étape 1. Chaque comportement est composé d'un certain nombre de réponses identifiées soit comme rawlsiennes, soit comme égalitaristes, soit comme utilitaristes. Les colonnes 2 à 4 renvoient au nombre de réponses qui ont été données pour chaque distribution par chacun des individus types, ce nombre étant centré et réduit autour de la moyenne et de la variance de chaque distribution. Finalement, la colonne 5 renvoie au résultat de l'ACM.

Ainsi, seul le comportement type numéro 8 reste indéterminé à la suite de l'ACM. Ce comportement peut à la fois être un comportement rawlsien et un comportement égalitariste.

\section{Annexe 2 : Test de Mann Whitney de différence de choix par catégorie de sujets}

Tableau A3. Test de Mann Whitney de différence de choix par catégorie de sujets

\begin{tabular}{cccc}
\hline $\begin{array}{c}\text { Choix de la } \\
\text { société } A\end{array}$ & Rawlsiens & Utilitaristes & Autres \\
\cline { 2 - 4 } Égalitaristes & -1.239 & $-0,842$ & 0,809 \\
& $(0,2152)$ & $(0,3998)$ & $(0,4185)$ \\
Rawlsiens & - & 0,484 & $-1,914$ \\
& & $(0,6282)$ & $(0,0556)$ \\
Utilitaristes & - & - & -1.586 \\
& & & $(0,1128)$ \\
\hline
\end{tabular}

Note : Le tableau présent la statistique de test $\mathrm{Z}$ ainsi que la $\mathrm{p}$-valeur entre parenthèses.

\section{Annexe 3 : Régressions}

\begin{tabular}{ccc}
\hline & MCO & Probit \\
Variables dépendantes : & \# de choix A & Choix A \\
Préférences sociales : & $(1)$ & $(2)$ \\
Constante (Egalitaristes) & -0.790 & $-2.034^{* * *}$ \\
Rawlsiens & $(1.348)$ & $(0.657)$ \\
& $0.613^{* *}$ & $0.419^{* * *}$ \\
& $(0.270)$ & $(0.155)$ \\
\hline
\end{tabular}




\begin{tabular}{ccc}
\hline Utilitaristes & $0.448^{*}$ & $0.321^{* *}$ \\
& $(0.268)$ & $(0.159)$ \\
Autres & 0.023 & 0.024 \\
& $(0.370)$ & $(0.200)$
\end{tabular}

Caractéristiques démographiques:

$\begin{array}{ccc}\text { Age } & 0.049^{* *} & 0.040^{* * *} \\ \text { Male } & (0.023) & (0.015) \\ & 0.509^{* *} & 0.370^{* *} \\ \text { Orientation politique à droite } & (0.219) & (0.150) \\ & -0,000 & -0,000 \\ & (0,005) & (0,003)\end{array}$

Préférences pour le risque

Éviter le risque (niveau 0) à cherche le risque (niveau 10)

\begin{tabular}{|c|c|c|}
\hline \multirow[t]{2}{*}{ Niveau 1} & 0.145 & 0.066 \\
\hline & (1.114) & $(0.369)$ \\
\hline \multirow[t]{2}{*}{ Niveau 2} & 0.357 & 0.241 \\
\hline & $(1.005)$ & $(0.179)$ \\
\hline \multirow[t]{2}{*}{ Niveau 3} & 0.768 & $0.496 * *$ \\
\hline & $(0.979)$ & $(0.250)$ \\
\hline \multirow[t]{2}{*}{ Niveau 4} & 0.239 & 0.133 \\
\hline & $(0.976)$ & $(0.338)$ \\
\hline \multirow[t]{2}{*}{ Niveau 5} & 0.641 & 0.421 \\
\hline & (1.020) & $(0.292)$ \\
\hline \multirow[t]{2}{*}{ Niveau 6} & 1.388 & $0.921 * * *$ \\
\hline & $(0.953)$ & $(0.242)$ \\
\hline \multirow[t]{2}{*}{ Niveau 7} & 0.777 & $0.526 * *$ \\
\hline & $(0.960)$ & $(0.234)$ \\
\hline \multirow[t]{2}{*}{ Niveau 8} & 1.032 & $0.673 * * *$ \\
\hline & $(0.954)$ & $(0.207)$ \\
\hline Niveau 9 & 1.027 & $0.681 * *$ \\
\hline
\end{tabular}




Niveau $10 \quad 1.209 \quad 0.784^{* *}$

$(1.055) \quad(0.316)$

Questions relatives à la PI :

\begin{tabular}{ccc} 
Q5 : « Déjà téléchargé illégalement » & -0.027 & -0.038 \\
Q2 : «La PI est justifiée » & $(0.267)$ & $(0.155)$ \\
Q1 : « Durée de la PI + longue » & 0.671 & $0.463^{* * *}$ \\
& $(0.436)$ & $(0.152$ \\
\hline $\mathrm{N}$ & $-0.212^{* *}$ & $-0.145^{* * *}$ \\
\hline & $(0.089)$ & $(0.052)$ \\
\hline
\end{tabular}

Annexe 3 : Test de Mann Whitney bilatéral entre les sujets utilitaristes par rapport aux sujets Rawlsiens à la question Q1

\begin{tabular}{lr}
\hline Utilitaristes & 368,000 \\
Espérance & 528,000 \\
Variance (Utilitaristes) & 5397,912 \\
p-value (bilatérale) & 0,031 \\
Alpha & 0,05 \\
\hline La p-value est calculée suivant une méthode exacte.
\end{tabular}

\section{Annexe 4 : Questionnaire}

Q1 : En France le droit d'auteur dur tout au long de la vie de l'auteur +70 ans après sa mort et le brevet dure 20 années. Pensez-vous que cette durée de protection doive être augmentée ou diminuée?

Notez votre réponse sur une échelle de -2 à $+2,-2$ indiquant qu'il faudrait beaucoup réduire la durée et +2 qu'il faudrait l'augmenter de beaucoup.

Q2 : Pensez-vous que la propriété intellectuelle soit justifiée?

1. Oui

2. Non 
Q3 : Si vous avez répondu oui à la question précédente, classez par ordre d'importance les trois justifications suivantes ( 1 indiquant que c'est celle qui vous semble la plus importante, 3 la moins importante) :

- La propriété intellectuelle est justifiée car elle permet d'encourager la création de nouvelles œuvres utiles à la société.

- La propriété intellectuelle est justifiée car elle protège le droit fondamental du créateur à s'approprier le résultat de son activité créative.

- La propriété intellectuelle est justifiée car elle permet de lutter contre le plagiat, c'està-dire contre le fait qu'une personne puisse revendiquer la paternité de l'œuvre/invention à la place de son créateur authentique.

Q4 : Y aurait-il d'autres raisons de justifier la propriété intellectuelle à vos yeux ? Si oui, laquelle (lesquelles)?

1. Oui

2. Non

Q5 : Avez-vous déjà téléchargé des contenus protégés par la propriété intellectuelle (textes/audio/vidéos, logiciels etc.) via un site web ou un logiciel de pairs à pairs ?

1. Oui

2. Non

Q6 : Si vous l'avez-fait pourquoi ?

1. J'ai téléchargé des contenus protégés car je ne parvenais pas à me les procurer de façon légale.

2. J'ai téléchargé des contenus protégés car il était trop cher de me les procurer de façon légale.

3. Autre, précisez :

Q7 : Avez-vous déjà produit des œuvres (ex: musique, dessins, romans, etc.) ou des inventions protégées par le droit de la propriété intellectuelle?

1. Oui

2. Non

Année de naissance :

Quel est votre sexe?

- Homme

- Femme

Quel est le revenu mensuel de votre foyer fiscal ?

- Moins de $799 €$

- De $800 €$ à $1199 €$ 
- De $1200 €$ à $1599 €$

- De $1600 €$ à $1999 €$

- De $2000 €$ à $2499 €$

- De 2500 à $2999 €$

- Plus de $3000 €$

Quel est votre plus haut diplôme obtenu ?

- Brevet

- Baccalauréat

- Licence

- Master

- Doctorat

Quel type d'étude avez-vous poursuivi ou êtes-vous en train de poursuivre actuellement ?

- Droit

- Economie, gestion

- Langues

- Sciences humaines et sociales (histoire, géographie, sociologie, arts etc.)

- Sciences (mathématique, physique/chimie, biologie, informatique etc.)

- Santé

- Autre : préciser

Votre nationalité

- Française

- Autre

Quelle est votre position religieuse?

- Athée/agnostique

- Catholique

- Protestant

- Orthodoxe

- Musulman

- Juif

- Hndouiste

- Bouddhiste

- Autre 
De quelle tendance politique êtes-vous le plus proche?

[Segment gauche-droite avec curseur]

Etes-vous généralement une personne qui prend des risques ou essayez-vous de les éviter ? Veuilez cocher une case sur l'échelle ci-dessous, où 0 signifie «peur du risque » et 10 signifie «prêt à prendre des risques »

[Segment avec échelle de 0 à 10]

Les personnes peuvent se comporter de façon différente selon les situations. Comment évaluez-vous votre disposition à prendre des risques dans les situations suivantes ?

[Segment avec échelle de 0 à 10]

- En conduisant?

- Dans les investissements financiers ?

- Pendant les loisirs et le sport ?

- Dans le travail?

- Avec votre santé ?

- Dans vos relations avec les autres ?

Veuillez considérer ce que vous feriez dans la situation suivante : Imaginez que vous avez gagné 100000 euros à la loterie. Presque immédiatement après avoir récupéré vos gains, vous recevez l'offre financière suivante de la part d'une banque réputée, les conditions sont les suivantes : il y a une chance de doubler l'argent dans deux ans. Il est également possible que vous perdiez la moitié du montant investi. Vous avez la possibilité d'investir tout l'argent que vous avez, une partie de cet argent ou de refuser l'offre.

Quel part de votre gain à la loterie êtes-vous prêt à investir dans cet investissement lucratif mais néanmoins risqué ?

$\begin{array}{lllll}20000 & 40000 & 60000 & 80000 \quad 100000 \quad \text { Rien je déclinerais }\end{array}$

l'offre 